\title{
Cadmium-Free and Efficient Type-II InP/ZnO/ZnS Quantum Dots and Their Application for LEDs
}

Guncem Ozgun Eren, Sadra Sadeghi, Houman Bahmani Jalali, Maximilian Ritter, Mertcan Han, Isinsu Baylam, Rustamzhon Melikov, Asim Onal, Fatma Oz, Mehmet Sahin, Cleva W. Ow-Yang, Alphan Sennaroglu, Rainer T. Lechner, and Sedat Nizamoglu*

Cite This: ACS Appl. Mater. Interfaces 2021, 13, 32022-32030

Read Online

ACCESS | Llll Metrics \& More | 回 Article Recommendations ｜（s Supporting Information

ABSTRACT: It is a generally accepted perspective that type-II nanocrystal quantum dots (QDs) have low quantum yield due to the separation of the electron and hole wavefunctions. Recently, high quantum yield levels were reported for cadmium-based typeII QDs. Hence, the quest for finding non-toxic and efficient type-II QDs is continuing. Herein, we demonstrate environmentally benign type-II $\mathrm{InP} / \mathrm{ZnO} / \mathrm{ZnS}$ core/shell/shell QDs that reach a high quantum yield of $\sim 91 \%$. For this, $\mathrm{ZnO}$ layer was grown on core InP QDs by thermal decomposition, which was followed by a $\mathrm{ZnS}$ layer via successive ionic layer adsorption. The small-angle $\mathrm{X}$ ray scattering shows that spherical $\mathrm{InP}$ core and $\mathrm{InP} / \mathrm{ZnO}$ core/ shell QDs turn into elliptical particles with the growth of the $\mathrm{ZnS}$

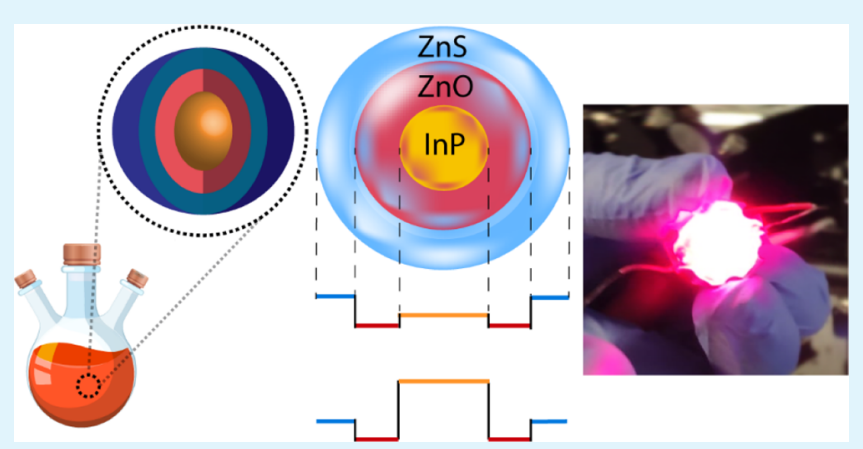
shell. To conserve the quantum efficiency of QDs in device architectures, $\mathrm{InP} / \mathrm{ZnO} / \mathrm{ZnS}$ QDs were integrated in the liquid state on blue light-emitting diodes (LEDs) as down-converters that led to an external quantum efficiency of $9.4 \%$ and a power conversion efficiency of $6.8 \%$, respectively, which is the most efficient QD-LED using type-II QDs. This study pointed out that cadmium-free type-II QDs can reach high efficiency levels, which can stimulate novel forms of devices and nanomaterials for bioimaging, display, and lighting.

KEYWORDS: indium phosphide, quantum dots, type-II band alignment, liquid LED, color conversion

\section{INTRODUCTION}

Semiconductor nanocrystal (NC) quantum dots (QDs) have attracted significant attention for light-generating devices such as luminescent solar concentrators, ${ }^{1-3}$ light-emitting diodes (LEDs), ${ }^{4-7}$ and lasers. ${ }^{8,9}$ They show advantageous properties such as tunable emission color due to the quantum confinement effect, high photochemical stability, and solution processability. ${ }^{10-13}$ Especially, high photoluminescence quantum yield (PLQY), defined as the ratio of the number of photons emitted to the total number of generated excitons, is a key figure of merit. To reach high PLQYs, type-I QDs with straddling alignment that localize the electron and hole inside the core are the most widely investigated heterostructures.

Alternatively, type-II QDs have conduction and valence band extrema in different materials, and it is a generally accepted perspective that type-II QDs result in low PLQYs. So far, a wide variety of type-II heterojunctions composed of $\mathrm{CdSe}, \mathrm{CdS}$, $\mathrm{CdTe}$, and $\mathrm{ZnTe}$ (e.g., $\mathrm{CdTe} / \mathrm{CdSe}$ and $\mathrm{ZnTe} / \mathrm{CdSe}$ ) have been examined. ${ }^{14-17}$ However, their PLQY remained low, which is attributed to the spatial separation of the electrons and holes that leads to the increase in the nonradiative recombination rates and decrease in PLQY. Recently, high PLQY values of $61,{ }^{18} 68,{ }^{19}$ and $88 \%{ }^{20}$ were achieved with $\mathrm{ZnSe} /$
$\mathrm{CdS} / \mathrm{ZnS}, \mathrm{CdSe} / \mathrm{CdTe}$, and $\mathrm{Cd}_{x} \mathrm{Zn}_{1-x} \mathrm{~S} / \mathrm{ZnSe} / \mathrm{ZnS}$ type-II QDs, respectively. However, the aforementioned type-II QDs have highly toxic heavy metal of cadmium. ${ }^{21-23}$ Alternatively, there were also a few cadmium-free QDs (e.g., ZnTe/ZnSe) investigated, but their PLQY reached up to $36 \% .^{24,25}$ Therefore, the quest for finding efficient and Cd-free type-II QDs, which can find widespread use to form environmentally benign lightgenerating devices and biocompatible luminescent markers, is continuing.

In this work, we demonstrate $\mathrm{InP} / \mathrm{ZnO} / \mathrm{ZnS}$ core/shell/shell QDs, which have type-II staggered line-up heterojunctions. We synthesized the $\mathrm{ZnO}$ shell with thermal decomposition and grow subsequent multiple $\mathrm{ZnS}$ shells with the Successive Ionic Layer Adsorption (SILAR) method. We optimized the $\mathrm{ZnO}$ and $\mathrm{ZnS}$ shell thickness that led to a high efficiency PLQY of $90.8 \%$.

Received: May 2, 2021

Accepted: June 17, 2021

Published: July 1, 2021 
a

b

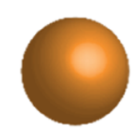

$\ln P$

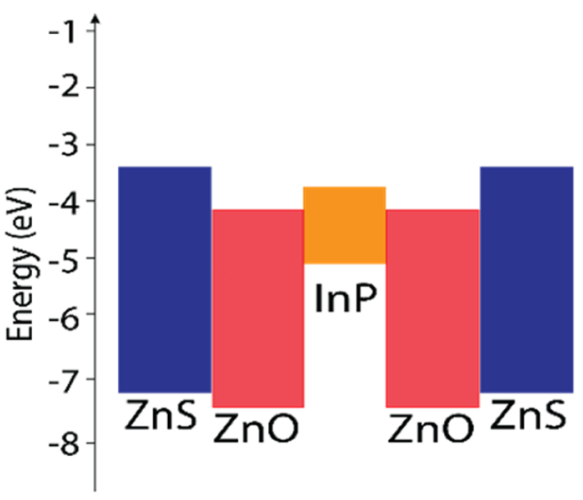

c

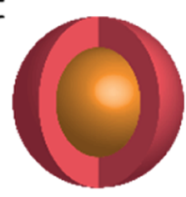

$\ln \mathrm{P} / \mathrm{ZnO}$ d

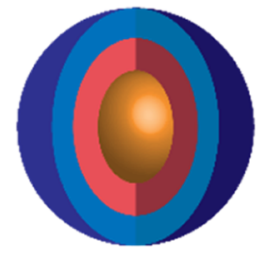

$\mathrm{InP} / \mathrm{ZnO} / \mathrm{ZnS}$
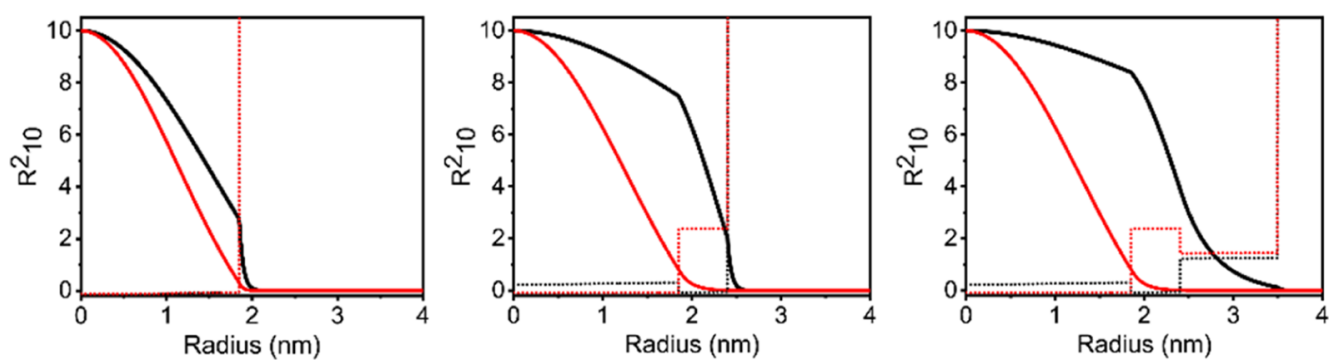

Figure 1. (a) Energy band diagram of $\mathrm{InP} / \mathrm{ZnO} / \mathrm{ZnS}$ core/shell/shell QDs. Quantum mechanical simulations of (b) InP core, (c) InP/ZnO core/ shell, and (d) InP/ZnO/ZnS core/shell/shell QDs. Black lines correspond to the radial probability distribution of electrons, while red lines show the radial probability distribution of holes. Black and red dashed lines correspond to confinement potential profile for electrons and holes, respectively.

Moreover, QDs were integrated in the liquid state on blue LEDs and QD-LEDs, demonstrating an external quantum efficiency (EQE) and a power conversion efficiency (PCE) of 9.4 and $6.6 \%$, respectively.

\section{RESULTS AND DISCUSSION}

Synthesis of InP/ZnO/ZnS Core/Shell/Shell QDs. InP core QDs were synthesized via a hot-injection method. ${ }^{1,26,27}$ For that, zinc undecylenate was used to passivate InP cationic dangling bonds and decrease trap states, ${ }^{26,28}$ and oleylamine $(\mathrm{OAM})$ and oleic acid (OA) were used as stabilizing agents. In brief, indium chloride $\left(\mathrm{InCl}_{3}\right), \mathrm{OAM}, \mathrm{OA}$, and zinc undecylenate were dissolved in the 1-octadecene (ODE) solvent in a glovebox at room temperature. After degassing and heating to $200{ }^{\circ} \mathrm{C}$, tris (trimethylsilyl)phosphine $\left[\mathrm{P}(\mathrm{TMS})_{3}\right]$ was injected into the reaction mixture to initiate the nucleation and growth. To generate a type-II band alignment, the $\mathrm{ZnO}$ shell was selected; by considering the successful growth of CdTe/ $\mathrm{ZnSe}$ core/shell QDs with a lattice mismatch of $14 \%,{ }^{29} \mathrm{ZnO}$ growth on $\mathrm{InP}$ with a lattice mismatch of $11 \%$ is also feasible. ${ }^{1,24,30}$ In this regard, $\mathrm{ZnO}$ was grown on the InP core by thermal decomposition of zinc acetylacetonate [ $\mathrm{Zn}$ $\left.(\text { acac })_{2}\right]^{31,32}$ During the synthesis, OAM was used as the stabilizing agent in order to hinder $\mathrm{ZnO}$ aggregation. ${ }^{33}$ Furthermore, different amounts of $\mathrm{ZnO}$ precursor solution was added for reaching the highest PLQY (see Table S1). To further increase the PLQY, we performed $\mathrm{ZnS}$ growth on the $\mathrm{InP} / \mathrm{ZnO}$ core/shell structure using a SILAR technique. For that, the reaction solution of $\mathrm{InP} / \mathrm{ZnO}$ was cooled to $170{ }^{\circ} \mathrm{C}$, zinc stearate-ODE $\left[\mathrm{Zn}(\mathrm{St})_{2}-\mathrm{ODE}\right]$ and sulfur-trioctylphosphine (S-TOP) stock solutions were added to reaction solution, and temperature was increased to $250{ }^{\circ} \mathrm{C}$ for $30 \mathrm{~min}$. Similarly, to grow additional $\mathrm{ZnS}$ shells, the temperature followed the same thermal cycling.

Quantum Mechanical Calculations and Optical Analysis. To understand the optical properties of the synthesized QDs, we calculated their quantum mechanical properties by selfconsistently solving Poisson-Schrödinger equations in the effective mass approximation (Figure $1 b-d$ ) and correlated them with the optical measurements (Figure 2). ${ }^{24}$ InP core QD has type-I band alignment that led the confinement of electrons and holes in the same spatial core region with a high overlap of the wavefunctions of $\sim 90 \%$ (Figure $1 \mathrm{~b}$ ). After $\mathrm{ZnO}$ shell growth, the band alignment of the nanostructure transit from type-I to type-II, and while hole wavefunction is confined completely in the core region like in $\mathrm{InP}$ core $\mathrm{QD}$, electron wavefunction expands toward the $\mathrm{ZnO}$ shell that reduces the wavefunction overlap to $60 \%$ (Figure 1c). ${ }^{34}$ Since the delocalization of the electron decreases the confinement energy level within the conduction band and the exciton binding energy, the photo- 

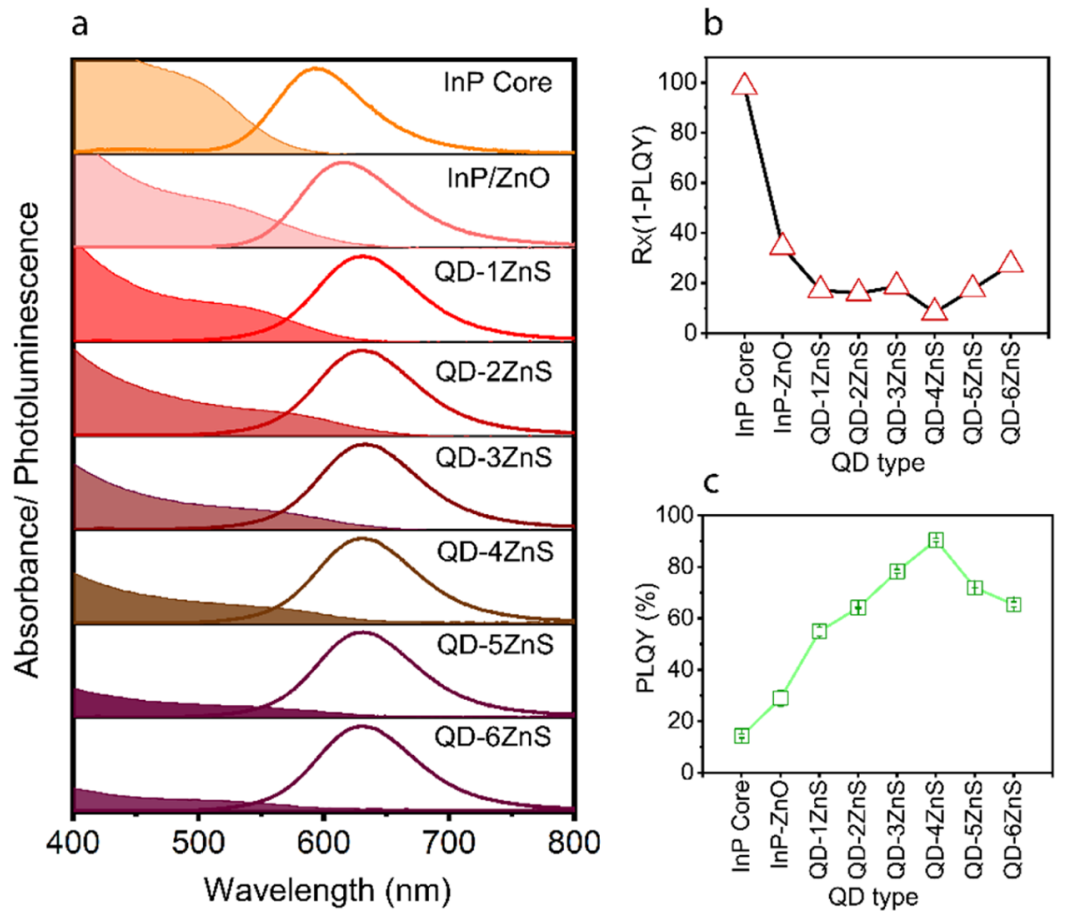

Figure 2. (a) Absorbance and $\mathrm{PL}$ spectra of $\mathrm{InP}, \mathrm{InP} / \mathrm{ZnO}$, and $\mathrm{InP} / \mathrm{ZnO} / \mathrm{ZnS}$ QDs with multiple shells. Here, QD refers to the $\mathrm{InP} / \mathrm{ZnO}$ core/shell QDs and \#ZnS refers to the number of SILAR growth cycles. (b) Normalized quenching factor of the QD-4ZnS core/shell NCs calculated by multiplication of reabsorption R and (1-PLQY). (c) PLQY (\%) of QDs in hexane. Errors bars represent the standard deviation from three measurements.

luminescence peak red-shifted from 585 to $613 \mathrm{~nm}$, while the full width at half-maximum (fwhm) was maintained at $\sim 74-75 \mathrm{~nm}$ for core and core/shell nanostructures. We investigated the $\mathrm{PLQY}$ of $\mathrm{ZnO}$ shell growth under different $\mathrm{ZnO}$ molar ratios, PLQY reached $25.8 \%$ (Figure 2a and see Supporting Information, Table S1).

Afterward to reach higher PLQYs, a variety of $\mathrm{ZnS}$ shell thickness was investigated. From 1 to 4 cycles of $\mathrm{ZnS}$ shell growth, the PLQY increased due to effective confinement of electron-hole pairs in the $\mathrm{InP}-\mathrm{ZnO}$ nanostructure and surface passivation (Figure 2c). ${ }^{35}$ After the fourth growth cycle of $\mathrm{ZnS}$, PLQY of $\mathrm{InP} / \mathrm{ZnO} / 4 \mathrm{ZnS} \mathrm{NCs}$ (QD-4ZnS NCs) started to decrease, possibly due to higher interfacial strain caused by dislocations. ${ }^{29,36}$ By the fourth $\mathrm{ZnS}$ shell, PLQY reached the maximum level of $90.8 \%$. QD-4ZnS showed a PL peak at 618 $\mathrm{nm}$, which is slightly red-shifted $(5 \mathrm{~nm})$ with respect to $\mathrm{InP} /$ $\mathrm{ZnO}$ QDs due to the lower wavefunction overlap of $49 \%$. We also investigated the effect of $\mathrm{ZnO}$ and $\mathrm{ZnS}$ shell growth on the normalized quenching factor $[\text { reabsorption } \times(1-\mathrm{PLQY})]^{37}$ for core and core/shell structures to select the appropriate nanostructure (Figure $2 \mathrm{~b}$ ). After $\mathrm{ZnO}$ shelling, due to red shifting of the PL peak, quenching factor decreased, and the minimum value is observed at QD-4ZnS NCs.

Structural Analysis. To understand the size and shape of the QDs, we performed small-angle X-ray scattering (SAXS) measurements in ethanol ${ }^{38}$ by probing up to $10^{6}$ individual particles. The scattering data after subtracting the ethanol background are shown in Figure S1, which are plotted versus the reciprocal scattering vector $q$. As shown in Figure 3a, we plotted instead of the reciprocal form factor $P(q)$ directly its Fourier transform, the pair distance distribution function (PDDF) in real space. ${ }^{38,39}$ The PDDF is the distribution of all distances within all single particles of the probed sample volume, which also contains the mean particle dimensions of the whole ensemble. From the maximum dimension found within the PDDF, we derive the maximum dimension found within the particle ensemble. The bell-like PDDFs of the InP-core and the $\mathrm{InP} / \mathrm{ZnO}$ core/shell QDs indicate spherical QD shapes, whereas the asymmetric shape of the QD-4ZnS-PDDF is a hint for an elongated mean shape. The change from a spherical shape for the InP and InP/ZnO QDs to a more elliptical one for the QD-4ZnS NCs can also be seen in the fact that only the first two SAXS curves could be fitted with monomodal distributions of spheres, as shown in Figure $3 \mathrm{~b}$. In these distributions, we derive from the peak positions directly the mean radii and hence the mean diameters of the QDs, from the widths of the size distributions. For the InP-core, we get a sphere diameter of $3.6 \pm$ $0.8 \mathrm{~nm}$ and for the $\mathrm{InP} / \mathrm{ZnO}$ core/shell QDs $4.4 \pm 0.9 \mathrm{~nm}$ with size distributions between 9 and $10 \%$, which is in excellent accordance with the transmission electron microscopy (TEM) data shown in the inset of Figure 3c,d. The asymmetric PDDF of QD-4ZnS NCs, however, indicates a prolate like mean particle shape. ${ }^{40}$ The short diameter is only a bit larger than the spherical diameter of $4.4 \mathrm{~nm}$ found for the $\mathrm{InP} / \mathrm{ZnO} \mathrm{NCs}$ as can be deduced from the slightly shifted peak of the QD-4ZnS-PDDF with respect to $\mathrm{InP} / \mathrm{ZnO}-\mathrm{PDDF}$ (see Figure 3a). The long axis is derived from the long tail within the PDDF, from which we deduce maximum values between 9 and $10 \mathrm{~nm}$. Most of the QDs within the ensemble depict long-axis values between 6 and $7 \mathrm{~nm}$, which is within the broader mean size distribution found by TEM with a maximum at $7.02 \pm 0.9 \mathrm{~nm}$ (Figure 3e). The elongated shape of the QD-4ZnS NCs together with their chainlike fractal aggregation (see Figure S1) can be explained by a slightly inhomogeneous growth of the $\mathrm{ZnS}$ shell, resulting in an overall prolate like NC shape. This kind of elliptical NC shape can be found also for other core/shell NC systems with thick shells, where the shell growth along specific crystallographic directions is energetically favored. ${ }^{41}$ The ellipsoidal shape of 
a

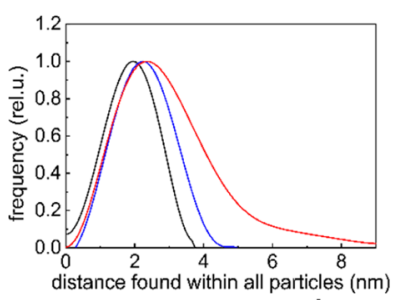

$$
\text { c }
$$

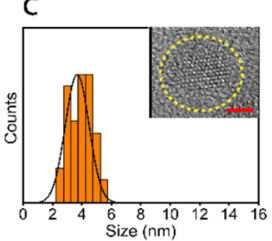

d

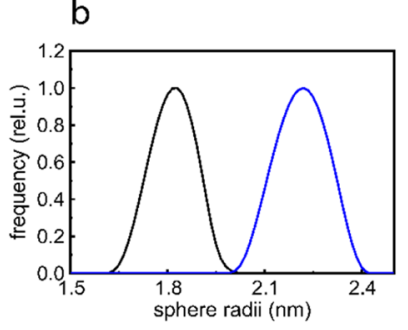
e
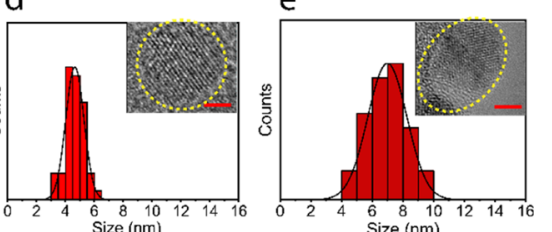

$f$
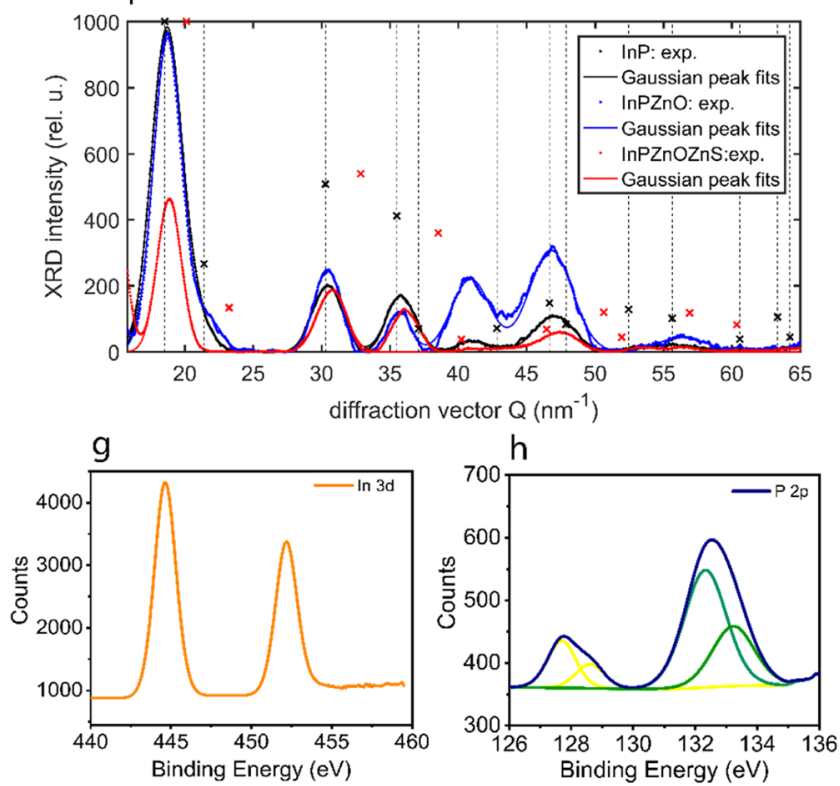

Figure 3. (a) PDDFs of the InP core (black), InP/ZnO core/shell QD (blue), and QD-4ZnS core/shell/shell NCs (red), as resulting from the $P(q)$-curves shown in Figure S1b. (b) Distributions of spheres that can describe the measured SAXS curves of the $\mathrm{InP}$ core and $\mathrm{InP} / \mathrm{ZnO}$ core/shell QDs. Size distribution histograms of the (c) InP core (inset: TEM image, scale bar is $2 \mathrm{~nm}$ ), (d) InP/ZnO core/shell (inset: TEM image, scale bar is $2 \mathrm{~nm}$ ), and (e) QD- $4 \mathrm{ZnS}$ core/shell/shell NCs (inset: TEM image, scale bar is $2 \mathrm{~nm}$ ) by high-resolution TEM measurements. Total number of 200 QDs are counted for size distribution. (f) $\mathrm{XRD}$ of $\mathrm{InP}, \mathrm{InP} / \mathrm{ZnO}$, and $\mathrm{InP} / \mathrm{ZnO} / 4 \mathrm{ZnS}$ core/ shell/shell NCs. XPS analysis of InP core QDs, (g) In $3 \mathrm{~d}$ spectrum and (h) P $2 \mathrm{p}$ spectrum.

nanoparticles can potentially lead to radiation polarization ${ }^{42}$ and additionally allowed transitions forbidden in spherical QDs. ${ }^{43}$

The crystal structure of the resulting QDs was investigated by analyzing the XRD patterns (Figures $3 \mathrm{f}$ and S2). The black vertical dashed lines and black crosses mark the positions of InP bulk peaks (ICDD 00-032-0452), the blue crosses of hexagonal $\mathrm{ZnO}$ (ICDD 00-036-1451), and the red crosses mark the positions of the cubic sphalerite phase of $\mathrm{ZnS}(a=5.4093 \AA$ ICDD 00-65-0309), respectively. From the only core QDs, we observe that the crystal structure corresponds to zinc blende ( $a=$ $5.869 \AA$, ICDD 00-32-0452). For InP/ZnO NCs, we still observed the InP zinc blende crystal structure, but due to the thin $\mathrm{ZnO}$ shell $(\sim 0.2-0.3 \mathrm{~nm})$, its diffraction peak (ICDD 00-
036-1451) cannot be detected. This is not surprising as only one monolayer (ML) shell material on a crystalline core can grow epitaxially by maintaining the lattice constant of the core. ${ }^{44}$ For the $\mathrm{InP} / \mathrm{ZnO} / \mathrm{ZnS} \mathrm{NCs}$, a shift of the InP peaks occurred in the direction of the position of $\mathrm{ZnS}$ in the cubic sphalerite phase (ICDD 00-65-0309). This shift can be explained by the situation that the first MLs of the $\mathrm{ZnS}$ shell initially maintains epitaxial registry with the lattice parameter of $\mathrm{InP}$ and with subsequent $\mathrm{ML}$ of $\mathrm{ZnS}$, tends to reach its bulk lattice constant. ${ }^{44}$ Because of the cubic lattice constant of $\mathrm{ZnS}$ is smaller than that of $\mathrm{InP}$, the InP core is compressively strained, reflected by peak shifts to larger $Q$-values or diffraction angles (see Figure $3 \mathrm{f}$ ). Additionally, we observe different peak-shift values as well as peak width values for the InP core along different crystallographic directions, indicating that the influence of the $\mathrm{ZnS}$ shell on the InP core is not isotropic. This result is suggestive of asymmetric $\mathrm{ZnS}$ shell growth as already derived from SAXS analysis.

X-ray photoelectron spectroscopy (XPS) analysis was performed to confirm the elemental surface chemistry of the InP core, InP/ZnO core/shell, and QD- $4 \mathrm{ZnS}$ core/shell/shell NCs, respectively. For the analysis, all of the peaks have been spectrally corrected according to the carbon-1s standard peak. For the InP core QDs, the In $3 \mathrm{~d}$ spectrum exhibits two peaks located at $444.5 \mathrm{eV}\left(3 \mathrm{~d}_{5 / 2}\right)$ and $452.1 \mathrm{eV}\left(3 \mathrm{~d}_{3 / 2}\right)$ (Figure $\left.3 \mathrm{~g}, \mathrm{~h}\right)$, which can be assigned to InP. ${ }^{45-47}$ The P $2 \mathrm{p}$ spectrum shows two doublets, which are related to the two different chemical environments of the phosphorus atoms. The first pre-dominant doublet that occurs at $127.8-128.9 \mathrm{eV}\left(2 \mathrm{p}_{3 / 2}\right)$ is the characteristic peak for InP. ${ }^{48,49}$ The doublet in the 132.4$133.4 \mathrm{eV}$ range is associated with the $\mathrm{P}$ atoms in an oxidized medium, most probably $\mathrm{InPO}_{X} \cdot{ }^{45,50}$ In the XPS spectra of InP/ $\mathrm{ZnO}$ core/shell QDs, the peaks corresponding to $\mathrm{Zn} 2 \mathrm{p}$ are observed which indicates the $\mathrm{Zn}^{2+}$ bound to oxygen in the $\mathrm{ZnO} .{ }^{51-53}$ The $\mathrm{Zn} 2 \mathrm{p}_{3 / 2}$ and $2 \mathrm{p}_{1 / 2}$ peaks are at 1022.09 and $1045.8 \mathrm{eV}$, respectively (Figure S3c). For the $\mathrm{InP} / \mathrm{ZnO}$ core/ shell QDs, the In $3 \mathrm{~d}$ peak shifts to higher binding energies located at $444.7 \mathrm{eV}\left(3 \mathrm{~d}_{5 / 2}\right)$ and $452.5 \mathrm{eV}\left(3 \mathrm{~d}_{3 / 2}\right)$, compared to the InP core QD (Figure S3a). Furthermore, shifting to higher binding energies located at 127.9 and $132.4 \mathrm{eV}$ also occurs in $\mathrm{P}$ $2 \mathrm{p}$ spectrum (Figure $\mathrm{S} 3 \mathrm{~b}$ ). Similarly, the $\mathrm{O} 1 \mathrm{~s}$ peak shifted to higher binding energy from 530.9 to $531.3 \mathrm{eV}$ after formation of the $\mathrm{ZnO}$ shell coating (Figure $\mathrm{S} 3 \mathrm{~d}$ ). The possible reason for this type of shifting is due to the additional oxidation process during the $\mathrm{ZnO}$ shelling procedure. ${ }^{45}$ For the QD-4ZnS core/shell/ shell NCs, the S 2 p spectrum exhibited two peaks located at $161.2-162.6 \mathrm{eV}$ (Figure S4d). It was previously reported that these binding energies are associated with $S^{2-}$ anions present in the $\mathrm{ZnS}{ }^{45,54,55}$ In addition, after the $\mathrm{ZnS}$ shell formation, In $3 \mathrm{~d}$ peaks shifted to lower binding energies located at $444.4 \mathrm{eV}$ $\left(3 \mathrm{~d}_{5 / 2}\right)$ and $452.0 \mathrm{eV}\left(3 \mathrm{~d}_{3 / 2}\right)$ (Figure S4a). Similarly, binding energies of $\mathrm{Zn} 2 \mathrm{p}$ and $\mathrm{O}$ 1s (Figure $\mathrm{S} 4 \mathrm{~b}, \mathrm{c}$ ) had also displaced to lower values after $\mathrm{ZnS}$ shelling due to decrease in the $\mathrm{InP}-\mathrm{O}$ bonds formed by oxidation. ${ }^{56}$

Ultrafast Decay Dynamics. The effect of shell growth on the ultrafast decay dynamics and the nonlinear absorption of QDs was further investigated using femtosecond pump-probe spectroscopy. ${ }^{37,57}$ In the experiments, the QD solutions (InP core, $\mathrm{InP} / \mathrm{ZnO}$ core/shell, and QD-4ZnS core/shell/shell NCs) were excited with $320 \mathrm{~nm}$, femtosecond pump pulses (incident fluence is $480 \mu \mathrm{J} \cdot \mathrm{cm}^{-2}$ ), and the difference between the pumped and unpumped absorbance $(\Delta A)$ of the QDs was measured over the $450-800 \mathrm{~nm}$ spectral range using a femtosecond visible continuum probe. Figure 4 summarizes the results of the 

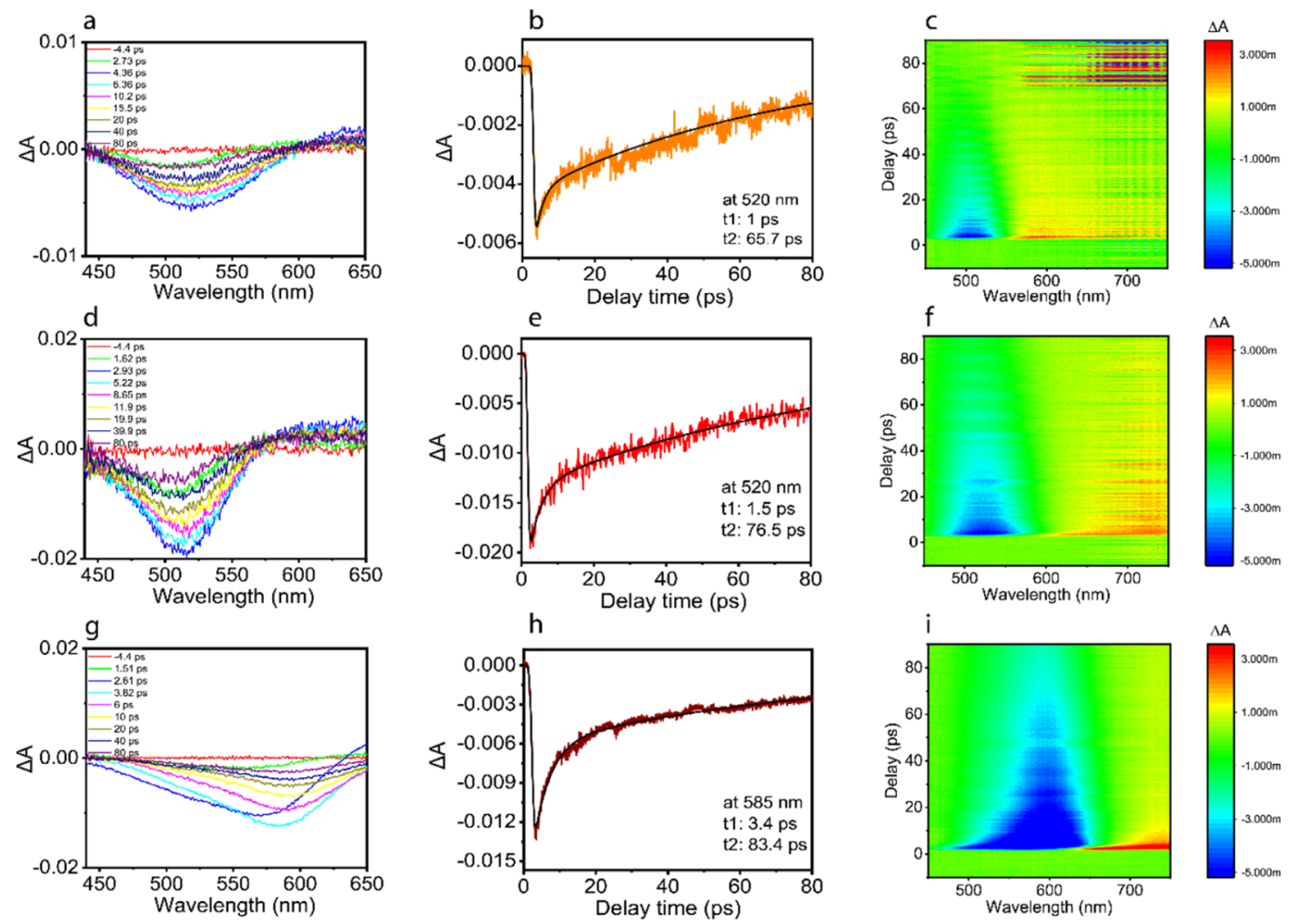

Figure 4. Measured $\Delta A$ spectrum, temporal evolution of $\Delta A$ at the wavelength of maximum bleaching, and two-dimensional variation of $\Delta A$ as a function of probe delay and wavelength for: $\mathrm{InP}$ core $(\mathrm{a}-\mathrm{c}), \mathrm{InP} / \mathrm{ZnO}(\mathrm{d}-\mathrm{f})$, and $\mathrm{QD}-4 \mathrm{ZnS}(\mathrm{g}-\mathrm{i}) \mathrm{NCs}$ for $\operatorname{InP}, \mathrm{InP} / \mathrm{ZnO}$, and QD-4ZnS NCs, respectively. Measured TA spectra of (a) InP core; (d) InP/ZnO and (g) QD-4ZnS at different probe delays. The excitation wavelength was $320 \mathrm{~nm}$. The measured peak wavelength of the nonlinear absorption changed and matched the bleach peak after the shell growth which can be explained by the reduction of the carrier-trap-induced stark effect. $(\mathrm{b}, \mathrm{e}, \mathrm{h})$ Measured and fitted temporal evolution of the carrier decay times at the probe wavelengths of 520 and $585 \mathrm{~nm}$. For the InP core, the first excitonic peak was at $520 \mathrm{~nm}$, and the $\tau_{1}$ and $\tau_{2}$ values were determined to be 1 and $65.7 \mathrm{ps}$. The $\mathrm{ZnO}$ shell growth reduced the trap states and hence, $\tau_{1}$ and $\tau_{2}$ increased to 1.5 and $76.5 \mathrm{ps}$, respectively. The multiple $\mathrm{ZnS}$ shell growth caused further trap passivation, resulting in short $\left(\tau_{1}\right)$ and long $\left(\tau_{2}\right)$ lifetimes of 3.4 and $84.3 \mathrm{ps}$, respectively. (c,f,i) Surface plots of the measured $\Delta A$ with respect to the probe delay and wavelength.

femtosecond transient absorption (TA) measurements. Measured $\Delta A$ spectrum, temporal evolution of $\Delta A$ at the wavelength of maximum bleaching, and two-dimensional variation of $\Delta A$ as a function of probe delay and wavelength are shown in Figure $4 \mathrm{a}-\mathrm{c}, \mathrm{d}-\mathrm{f}, \mathrm{g}-\mathrm{i}$, for InP, InP/ZnO, and QD-4ZnS NCs, respectively. The peak wavelength of the measured $\Delta A$ spectra of the QDs exhibited a red-shift as a function of the probe delay due to the intra-band decay of the excited carriers to the conduction band edge ${ }^{58}$ (Figure $4 \mathrm{a}, \mathrm{d}, \mathrm{g}$ ). The TA measurements showed that for the InP core, the steady-state absorption peak wavelength was at $490 \mathrm{~nm}$, and the peak wavelength of bleach was at $520 \mathrm{~nm}$ (Figure 4a). However, after the growth of $\mathrm{ZnO}$ or $\mathrm{ZnS}$ shell over InP core, the peak wavelengths of steady-state absorption and bleach became nearly equal (Figure $4 \mathrm{a}, \mathrm{d}, \mathrm{g}$ ), which can be explained based on the reduction of the biexciton and carrier-trap induced stark effect. ${ }^{59}$ The ultrafast temporal evolution of the carriers was further investigated for probe wavelengths at which the measured $\Delta A$ showed the maximum amount of bleach ( 520 and $585 \mathrm{~nm}$ ) for the $\mathrm{InP}, \mathrm{InP} / \mathrm{ZnO}$, and QD-4ZnS NCs. As can be seen in Figure 4b,e,h, following an initial rise, a biexponential decay occurred, which could be characterized by the decay times $\tau_{1}$ and $\tau_{2}{ }^{60,61}$ Here, the decay times $\tau_{1}$ and $\tau_{2}$ are due to shallow and deeper traps, which originate due to the $\mathrm{P}$ and In dangling bonds. ${ }^{62}$ The decay times of $\tau_{1}$ and $\tau_{2}$ for the InP, InP/ZnO, and QD-4ZnS NCs were determined by performing a biexponential fit to the measured ultrafast decay data. The fit results showed that $\tau_{1}$ and $\tau_{2}$ both increased from 1 to $1.5 \mathrm{ps}$ and from 65.7 to $76.5 \mathrm{ps}$, respectively, after the shell growth of $\mathrm{ZnO}$ on the InP core. Furthermore, for the case of QD-4ZnS NCs, $\tau_{1}$ and $\tau_{2}$ became 3.4 and $84.3 \mathrm{ps}$, respectively, indicating further passivation of the shallow and deeper traps as a result of shell growth..$^{59,63-65}$ Note that the measured $\Delta A$ did not vanish completely for the longest probe delay of 80 ps since the carrier recombination for the QDs occur over nanosecond time scales (Figure S5).

LED Applications. To prevent any efficiency drop due to the host material effect, we designed an architecture $^{66}$ that can maintain QD solution in the liquid state on top of the blue LED (Figure 5a). For that we used a transparent polymeric lens made of polydimethylsiloxane (PDMS) due to its scalable and low cost fabrication. ${ }^{67,68}$ We placed the lens on top of the blue LED chip and sealed the edges with the UV-curable polymer. After the curing process was finished, we injected red-emitting QDs using a microsyringe into the blue LED capped with a PDMS polymeric lens. When the blue LED is turned on, the blue electroluminescence generated from the blue LED die optically pumps the QDs in the solution state, which generates photoluminescence in the red spectral region (Figure 5a,b). To analyze the optical performance of the fabricated QD-LED, we injected the QD solution with different optical densities. While the optical density increases, red emission becomes stronger in comparison with the blue electroluminescence 


\section{a}

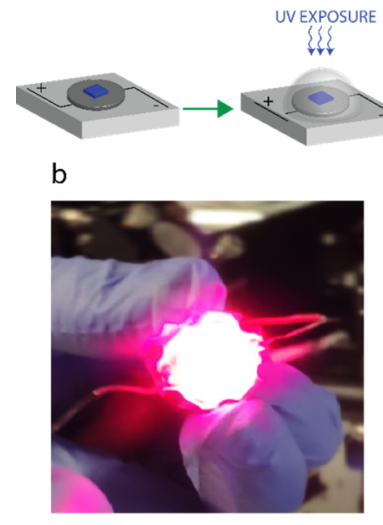

d

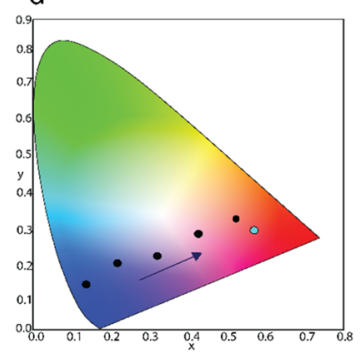

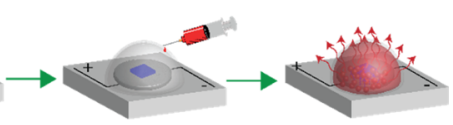

C

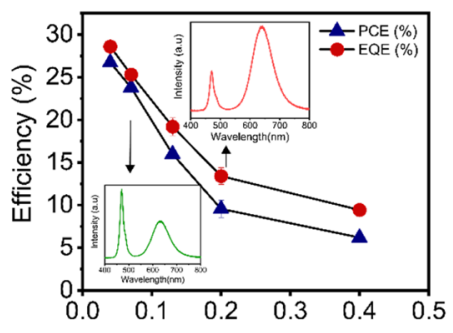

e

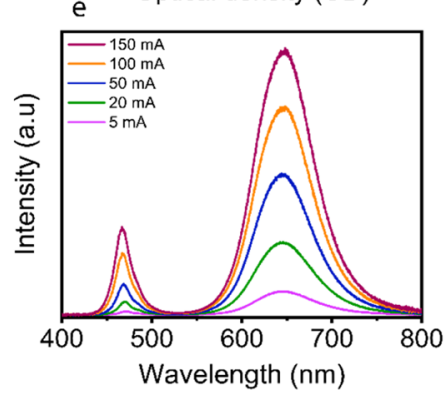

Figure 5. (a) Schematic of the QD-based LED fabrication. (b) Photograph of the LED when it is turned on. (c) PCE (\%) and EQE (\%) at different OD values. Inset: Intensity spectra of red-emitting QDbased LEDs at different optical densities of 0.07 and 0.2. (d) $(x, y)$ color coordinates of the QD-LEDs at different optical densities with an injection current of $10 \mathrm{~mA}$. (e) Intensity spectra of the red-emitting QD-based LED with an optical density of 0.4 at different current injection levels ranging from 5 to $150 \mathrm{~mA}$.

[Figure 5c]. Finally, the $(x, y)$ tristimulus coordinates reached the red region on the CIE chromaticity diagram by integrating QDs with an optical density of 0.4 (Figure $5 \mathrm{~d}$ ). The intensity spectra remained almost constant with a slight change in the PL peak position of $3 \mathrm{~nm}$, while the current injection level increases from 5 to $150 \mathrm{~mA}$ (Figure 5e). The EQE was measured as 28.6, 25.3, 19.2, 13.4, and 9.43\% for the optical densities of 0.04, 0.07, $0.13,0.20$, and 0.4 , respectively. Moreover, PCE is calculated by the relation of PCE $=\eta_{\text {ext. }} \lambda_{1} \cdot\left(\lambda_{2}\right)^{-1}$, 6 where $\lambda_{1}$ and $\lambda_{2}$ are the emission peak wavelength of the blue EL and emission peak wavelength of the QDs, respectively. PCE of LEDs corresponded to $21.62,18.85,13.99,9.85$, and $6.86 \%$ for the respective OD values, respectively.

Till date, in terms of type-II QD-based LED studies, Jin et al. synthesized green-emitting alloyed $\mathrm{Cd}_{x} \mathrm{Zn}_{1-x} \mathrm{~S} / \mathrm{ZnSe}$ type-II QDs, and these LED results demonstrated EQE of $8.78 \%{ }^{20}$ Moreover, Lin et al. reported deep-red LEDs based on CdTe/ CdSe core/shell QDs with a maximum EQE of $6.19 \% .{ }^{19}$ Instead of cadmium-based QDs, Karatum et al. integrated $\mathrm{InP} / \mathrm{ZnO}$ core/shell QDs as an emissive layer into LED applications, resulting in an EQE of $0.53 \% .{ }^{30}$ Comparatively, the LED using InP-based type-II QDs in this study reached the EQE level, approaching to $10 \%$ due to the high efficiency in synthesis batch and conserving their efficiency levels by direct integration in the liquid state.

Conclusions. In summary, we demonstrated cadmium-free and efficient type-II InP/ZnO/ZnS core/shell/shell QDs. For that, initially InP core QDs with emission in the red spectral region were synthesized using a hot injection technique. Then,

the sequential growth of $\mathrm{ZnO}$ and $\mathrm{ZnS}$ shells led to a high PLQY of $90.8 \%$. $\mathrm{ZnO}$ and $\mathrm{ZnS}$ shell formation were confirmed by optical analysis that is supported by quantum mechanical simulations. Moreover, SAXS measurements showed that the $\mathrm{ZnS}$ shell induced the transition of the NC ensemble from spherical to elliptical shapes. Finally, we integrated the core/ shell/shell QDs into LED die in the liquid form to reduce the host-material effect. The fabricated liquid-LED device demonstrated $9.4 \%$ of EQE and $6.8 \%$ of PCE, respectively, which is the most efficient type-II QD-based LED reported till date. Type-II QDs show high promise for future bioimaging, display, and laser applications.

\section{EXPERIMENTAL SECTION}

Chemicals. Zinc undecylenate (99\%), OA (99\%), OAM (99\%), ODE (90\%), indium(III) chloride ( $\left.\mathrm{InCl}_{3}\right)(99 \%), \mathrm{P}(\mathrm{TMS})_{3}(95 \%)$, $\mathrm{Zn}(\mathrm{St})_{2}$, TOP, sulfur $(\mathrm{S})$ (reagent grade, powder, purified by refining, -100 mesh particle size), and zinc acetylacetonate hydrate $\left[\mathrm{Zn}(\mathrm{acac})_{2}\right]$ (99.995\%) were purchased from Sigma-Aldrich. ODE was purified at $120^{\circ} \mathrm{C}$ by evacuating and refilling repeatedly with nitrogen for $1 \mathrm{~h}$. All the procedures were performed in a glovebox under a nitrogen atmosphere.

Synthesis of InP Core. First, $43 \mathrm{mg}$ of zinc undecylenate, $32 \mu \mathrm{L}$ of $\mathrm{OA}, 66 \mu \mathrm{L}$ of OAM, and $22 \mathrm{mg}$ of $\mathrm{InCl}_{3}$ were mixed in $3 \mathrm{~mL}$ of ODE and heated to $120^{\circ} \mathrm{C}$. The flask was degassed $20 \mathrm{~min}$ at $120^{\circ} \mathrm{C}$ and refilled with nitrogen. Afterward, it was heated to $200^{\circ} \mathrm{C}$, and $500 \mu \mathrm{L}$ of phosphine stock solution $\left[\mathrm{P}(\mathrm{TMS})_{3}\right.$-ODE $0.2 \mathrm{mmol} \mathrm{mL}^{-1}$ ] was rapidly injected and mixed $30 \mathrm{~min}$ at $200^{\circ} \mathrm{C}$. Then, the solution was cooled to $80^{\circ} \mathrm{C}$, and an aliquot was taken from the sample.

Synthesis of InP/ZnO and QD-4ZnS Core/Shell/Shell NCs. In order to prepare the $\mathrm{ZnO}$ stock solution, $245 \mu \mathrm{L}$ of OAM, $8 \mu \mathrm{L}$ of OA, and $6.5 \mathrm{mg}$ of $\mathrm{Zn}(\mathrm{acac})_{2}$ were mixed in $1.6 \mathrm{~mL}$ of ODE at $80^{\circ} \mathrm{C} .500 \mu \mathrm{L}$ of $\mathrm{ZnO}$ stock solution was added to $\mathrm{InP}$ core solution at $80{ }^{\circ} \mathrm{C}$ and heated to $250{ }^{\circ} \mathrm{C}$. It was mixed $30 \mathrm{~min}$ at this temperature and cooled to $170{ }^{\circ} \mathrm{C}$. For the zinc $(\mathrm{Zn})$ and sulfur $(\mathrm{S})$, precursor solutions were prepared separately. $510 \mathrm{mg}$ of $\mathrm{Zn}(\mathrm{St})_{2}$ and $27 \mathrm{mg}$ of $S$ powder were dissolved in $8 \mathrm{~mL}$ of ODE and $8 \mathrm{~mL}$ of TOP, respectively. It should be noted that for fully dissolving sulfur in TOP, we applied heat which was around $100^{\circ} \mathrm{C}$. After preparation of stock solutions, they were added to $\mathrm{InP} / \mathrm{ZnO}$ solution in a row and heated to $250^{\circ} \mathrm{C}$. The solution was mixed at $250{ }^{\circ} \mathrm{C}$ for $30 \mathrm{~min}$. For each shelling procedure, the same method was employed. The injection amounts of precursors are shown in Table S2.

Purification and Storage. After synthesis, QD solution was directly centrifuged at $9000 \mathrm{rpm}$ for $10 \mathrm{~min}$ two times. The precipitated part was removed from the QD solution, and ethanol was added to the solution until it became turbid and centrifuged again with same parameters. The precipitated QDs were dispersed in hexane and kept at $4{ }^{\circ} \mathrm{C}$ and covered with the aluminum foil.

Lens Making Procedure. In order to make hemispherical lens with outer diameter of $9 \mathrm{~nm}$ and inner diameter of $7 \mathrm{~nm}$, the PDMSSYLGARD 184 elastomer was mixed with the SYLGARD 184 curing agent with a ratio of 10:1, and then, the mixture was stirred vigorously until the bubbles appear. Afterward, to dispel the bubbles completely, the mixture was degassed $20 \mathrm{~min}$. The mixture was poured into the aluminum mold and cured at $70^{\circ} \mathrm{C} 1 \mathrm{~h}$. After curing, lens was taken out from the mold. The thickness of the as-prepared lens is $1 \mathrm{~mm}$.

Fabrication of LED Devices and Close-Packed LEDs. Before soldering of the electrical wires for connection to the voltage supply, the blue chip was mounted on board. Afterward, PDMS lens was attached on LEDs by using an NOA 68 UV-curable polymer. To cure LEDs with PDMS, lens were kept under UV radiation at $365 \mathrm{~nm}$ for $20 \mathrm{~min}$. To prevent any leakage from the PDMS lens, dripping of the curable polymer and keeping under radiation were performed two times.

LED Measurements. For LED measurements, an EP-B4040F-A3 InGaN GaN blue LED chip from Secol Company with an illumination wavelength at $465 \mathrm{~nm}$ was used. LED measurements were performed with a multi-port Ocean Optics integrating sphere. The detector was an 
Ocean Optics Torus with an optical resolution of $1.6 \mathrm{~nm}$ fwhm. The signal to noise ratio was 250:1. The EQE was calculated based on the literature, as mentioned above.

Structural and Optical Characterizations of QDs. Powder Xray diffraction (XRD) measurements of the $\mathrm{InP}, \mathrm{InP} / \mathrm{ZnO}$, and QD$4 \mathrm{ZnS}$ NCs were performed by a Bruker D8 eco X-ray diffractometer $\left(\lambda_{\mathrm{Cu} \mathrm{K} \alpha}=1.54 \AA\right.$ radiation $)$ with a scan speed of $0.14^{\circ} \mathrm{min}^{-1}$. Before XRD measurements, they were purified by three times to remove excess impurities. Then, the colloidal NC dispersion was drop-cast on a Simiscut wafer, a zero-background holder. XRD measurements were carried out at room temperature. Steady-state absorbance and PL measurements were performed using an Edinburgh Instruments FL-50 spectrofluorometer with an excitation wavelength of $375 \mathrm{~nm}$. QE measurements were carried out using an integrating sphere with an inner diameter of $150 \mathrm{~mm}$.

For TEM measurements, samples were deposited as $10 \mu \mathrm{L}$ of $1 \mathrm{mM}$ of $\mathrm{QD}$ in hexane solution on a copper support grid. TEM analysis was performed using a JEOL JEM-ARM200CFEG UHR microscope with a spherical aberration-corrected probe and equipped with a Gatan UltraScan camera model 994 US1000X.

Bright-field images were collected using an accelerating voltage of $200 \mathrm{keV}$. For the SAXS measurements about $100 \mu \mathrm{L}$ of NC-dispersions have been filled in quartz glass capillaries with $1.5 \mathrm{~mm}$ diameter and have been sealed with epoxy resin. The capillaries have been inserted into our laboratory SAXS system (Nanostar from Bruker AXS) and have been measured in vacuum to suppress air scattering. Twodimensional SAXS images have been recorded using $\mathrm{Cu} \mathrm{K} \alpha \mathrm{X}$-ray radiation with a wavelength of $1.5 \AA$ and a 2D Vantec detector. The 2D scattering patterns have been azimuthal integrated to obtain 1D scattering intensity $I(q)$ data as a function of the reciprocal scattering vector $q$. All $1 \mathrm{D}$ data have been corrected for the different X-ray transmission values.

Transient absorption (TA) studies were performed with an ultrafast pump-probe spectrometer to measure the change $\Delta A$ in the nonlinear absorbance spectrum ( $\Delta A=A_{\text {pumped }}-A_{\text {unpumped }} ; A_{\text {pumped }}=$ absorbance spectrum of the pumped sample and $A_{\text {unpumped }}=$ small-signal absorbance spectrum). Further details of the pump-probe setup and measurement procedure are described in ref 37 . Here, free charge carriers were excited with a $320 \mathrm{~nm}$ femtosecond pump pulse (fluence of $480 \mu \mathrm{J} \cdot \mathrm{cm}^{-2}$ ), and the resulting nonlinear absorbance change was measured with a femtosecond white-light probe in the $450-800 \mathrm{~nm}$ spectral window.

\section{ASSOCIATED CONTENT}

\section{(s) Supporting Information}

The Supporting Information is available free of charge at https://pubs.acs.org/doi/10.1021/acsami.1c08118.

PLQY values according to the injected $\mathrm{ZnO}$ precursor; zinc and sulfur precursor solution concentration for $\mathrm{ZnS}$ shells; SAXS data (symbols) and fits of InP core, InP/ $\mathrm{ZnO}$, and QD-4ZnS; XRD of InP core, $\mathrm{InP} / \mathrm{ZnO}$, and QD-4ZnS; XPS analysis of InP/ZnO QDs; XPS analysis of QD-4ZnS QDs; and surface plots of the measured TA spectra (PDF)

\section{AUTHOR INFORMATION}

\section{Corresponding Author}

Sedat Nizamoglu - Department of Biomedical Science and Engineering, Graduate School of Material Science and Engineering, Department of Electrical and Electronics Engineering, and Koc University Boron and Advanced Materials Application and Research Center, Koç University, Istanbul 34450, Turkey; @orcid.org/0000-0003-03945790; Email: snizamoglu@ku.edu.tr

\section{Authors}

Guncem Ozgun Eren - Department of Biomedical Science and Engineering, Koç University, Istanbul 34450, Turkey; (1) orcid.org/0000-0002-9297-9898

Sadra Sadeghi - Graduate School of Material Science and Engineering, Koç University, Istanbul 34450, Turkey; (1) orcid.org/0000-0002-8569-1626

Houman Bahmani Jalali - Department of Biomedical Science and Engineering, Koç University, Istanbul 34450, Turkey; (1) orcid.org/0000-0001-7212-9098

Maximilian Ritter - Institute of Physics, Montanuniversitaet Leoben, Leoben 8700, Austria

Mertcan Han - Department of Electrical and Electronics Engineering, Koç University, Istanbul 34450, Turkey; 잉 orcid.org/0000-0002-3543-5894

Isinsu Baylam - Koç University Surface Science and Technology Center (KUYTAM), Koç University, Istanbul 34450, Turkey; (1) orcid.org/0000-0003-2327-9035

Rustamzhon Melikov - Department of Electrical and Electronics Engineering, Koç University, Istanbul 34450, Turkey; 이이이.org/0000-0003-2214-7604

Asim Onal - Graduate School of Material Science and Engineering, Koç University, Istanbul 34450, Turkey

Fatma Oz-Department of Biomedical Science and Engineering, Koç University, Istanbul 34450, Turkey

Mehmet Sahin - Department of Nanotechnology Engineering, Abdullah Gul University, Kayseri 38080, Turkey; (i) orcid.org/0000-0002-9419-1711

Cleva W. Ow-Yang - SUNUM Nanotechnology Research and Application Center, Sabanci University, Istanbul 34956,

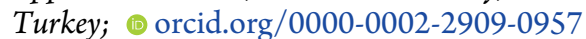

Alphan Sennaroglu - Koç University Surface Science and Technology Center (KUYTAM) and Laser Research Laboratory, Departments of Physics and Electrical-Electronics Engineering, Koç University, Istanbul 34450, Turkey; (1) orcid.org/0000-0003-4391-0189

Rainer T. Lechner - Institute of Physics, Montanuniversitaet

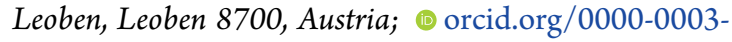
0038-1088

Complete contact information is available at: https://pubs.acs.org/10.1021/acsami.1c08118

\section{Notes}

The authors declare no competing financial interest.

\section{ACKNOWLEDGMENTS}

S.N. acknowledges support by the European Research Council (ERC) under the European Union's Horizon 2020 Research and Innovation Programme (grant agreement no. 639846). S.N. also acknowledges the support by the Turkish Academy of Sciences (TÜBA-GEBIP; The Young Scientist Award Program); the Science Academy of Turkey (BAGEP; The Young Scientist Award Program); and Bilim Kahramanları Derneği (The Young Scientist Award Program).

\section{REFERENCES}

(1) Sadeghi, S.; Bahmani Jalali, H.; Melikov, R.; Ganesh Kumar, B.; Mohammadi Aria, M.; Ow-Yang, C. W.; Nizamoglu, S. Stokes-shiftengineered indium phosphide quantum dots for efficient luminescent solar concentrators. ACS Appl. Mater. Interfaces 2018, 10, 1297512982. 
(2) Liu, G.; Mazzaro, R.; Wang, Y.; Zhao, H.; Vomiero, A. High efficiency sandwich structure luminescent solar concentrators based on colloidal quantum dots. Nano Energy 2019, 60, 119-126.

(3) Meinardi, F.; McDaniel, H.; Carulli, F.; Colombo, A.; Velizhanin, K. A.; Makarov, N. S.; Simonutti, R.; Klimov, V. I.; Brovelli, S. Highly efficient large-area colourless luminescent solar concentrators using heavy-metal-free colloidal quantum dots. Nat. Nanotechnol. 2015, 10, $878-885$.

(4) Rogach, A. L.; Gaponik, N.; Lupton, J. M.; Bertoni, C.; Gallardo, D. E.; Dunn, S.; Li Pira, N.; Paderi, M.; Repetto, P.; Romanov, S. G.; O'Dwyer, C.; Sotomayor Torres, C. M.; Eychmüller, A. Light-emitting diodes with semiconductor nanocrystals. Angew. Chem., Int. Ed. 2008, $47,6538-6549$.

(5) Shirasaki, Y.; Supran, G. J.; Bawendi, M. G.; Bulović, V. Emergence of colloidal quantum-dot light-emitting technologies. Nat. Photonics 2013, 7, 13.

(6) Won, Y.-H.; Cho, O.; Kim, T.; Chung, D.-Y.; Kim, T.; Chung, H.; Jang, H.; Lee, J.; Kim, D.; Jang, E. Highly efficient and stable InP/ZnSe/ $\mathrm{ZnS}$ quantum dot light-emitting diodes. Nature 2019, 575, 634-638.

(7) Sadeghi, S.; Mutcu, S. E.; Srivastava, S. B.; Aydindogan, G.; Caynak, S.; Karsl1, K.; Melikov, R.; Nizamoglu, S. High quality quantum dots polymeric films as color converters for smart phone display technology. Mater. Res. Express 2018, 6, 035015.

(8) Sabatini, R. P.; Bappi, G.; Bicanic, K. T.; Fan, F.; Hoogland, S.; Saidaminov, M. I.; Sagar, L. K.; Voznyy, O.; Sargent, E. H. Temperature-Induced Self-Compensating Defect Traps and Gain Thresholds in Colloidal Quantum Dots. ACS Nano 2019, 13, 89708976.

(9) Wang, Y.; Ta, V. D.; Leck, K. S.; Tan, B. H. I.; Wang, Z.; He, T.; Ohl, C.-D.; Demir, H. V.; Sun, H. Robust whispering-gallery-mode microbubble lasers from colloidal quantum dots. Nano Lett. 2017, 17, 2640-2646.

(10) Murray, C. B.; Norris, D. J.; Bawendi, M. G. Synthesis and characterization of nearly monodisperse $\mathrm{CdE}(\mathrm{E}=$ sulfur, selenium, tellurium) semiconductor nanocrystallites. J. Am. Chem. Soc. 1993, 115, 8706-8715.

(11) Yin, Y.; Talapin, D. The chemistry of functional nanomaterials. Chem. Soc. Rev. 2013, 42, 2484-2487.

(12) Dai, X.; Zhang, Z.; Jin, Y.; Niu, Y.; Cao, H.; Liang, X.; Chen, L.; Wang, J.; Peng, X. Solution-processed, high-performance light-emitting diodes based on quantum dots. Nature 2014, 515, 96-99.

(13) Han, M.; Jalali, H. B.; Yildiz, E.; Qureshi, M. H.; Şahin, A.; Nizamoglu, S. Photovoltaic neurointerface based on aluminum antimonide nanocrystals. Commun. Mater. 2021, 2, 19.

(14) Zhang, Y.; Li, Y.; Yan, X.-P. Photoactivated CdTe/CdSe quantum dots as a near infrared fluorescent probe for detecting biothiols in biological fluids. Anal. Chem. 2009, 81, 5001-5007.

(15) Gentle, C. M.; Wang, Y.; Haddock, T. N.; Dykstra, C. P.; van der Veen, R. M. Internal Atomic-Scale Structure Determination and Band Alignment of II-VI Quantum Dot Heterostructures. J. Phys. Chem. C 2020, 124, 3895-3904.

(16) Long, T.; Cao, J.; Jiang, Z.-J. Predictable spectroscopic properties of type-II ZnTe/CdSe nanocrystals and electron/hole quenching. Phys. Chem. Chem. Phys. 2019, 21, 5824-5833.

(17) Ca, N. X.; Hien, N. T.; Luyen, N. T.; Lien, V. T. K.; Thanh, L. D.; Do, P. V.; Bau, N. Q.; Pham, T. T. Photoluminescence properties of $\mathrm{CdTe} / \mathrm{CdTeSe} / \mathrm{CdSe}$ core/alloyed/shell type-II quantum dots. J. Alloys Compd. 2019, 787, 823-830.

(18) Tyrakowski, C. M.; Shamirian, A.; Rowland, C. E.; Shen, H.; Das, A.; Schaller, R. D.; Snee, P. T. Bright Type II Quantum Dots. Chem. Mater. 2015, 27, 7276-7281.

(19) Lin, Q.; Song, B.; Wang, H.; Zhang, F.; Chen, F.; Wang, L.; Li, L. S.; Guo, F.; Shen, H. High-efficiency deep-red quantum-dot lightemitting diodes with type-II CdSe/CdTe core/shell quantum dots as emissive layers. J. Phys. Chem. C 2016, 4, 7223-7229.

(20) Jin, X.; Li, H.; Huang, S.; Gu, X.; Shen, H.; Li, D.; Zhang, X.; Zhang, Q.; Li, F.; Li, Q. Bright alloy type-II quantum dots and their application to light-emitting diodes. J. Colloid Interface Sci. 2018, 510, 376-383.
(21) Chen, N.; He, Y.; Su, Y.; Li, X.; Huang, Q.; Wang, H.; Zhang, X.; Tai, R; Fan, C. The cytotoxicity of cadmium-based quantum dots. Biomaterials 2012, 33, 1238-1244.

(22) Derfus, A. M.; Chan, W. C. W.; Bhatia, S. N. Probing the cytotoxicity of semiconductor quantum dots. Nano Lett. 2004, 4, 1118.

(23) Kirchner, C.; Liedl, T.; Kudera, S.; Pellegrino, T.; Muñoz Javier, A.; Gaub, H. E.; Stölzle, S.; Fertig, N.; Parak, W. J. Cytotoxicity of colloidal CdSe and CdSe/ZnS nanoparticles. Nano Lett. 2005, 5, 331338.

(24) Bahmani Jalali, H.; Mohammadi Aria, M.; Dikbas, U. M.; Sadeghi, S.; Ganesh Kumar, B.; Sahin, M.; Kavakli, I. H.; Ow-Yang, C. W.; Nizamoglu, S. Effective neural photostimulation using indiumBased type-II quantum dots. ACS Nano 2018, 12, 8104-8114.

(25) Bang, J.; Park, J.; Lee, J. H.; Won, N.; Nam, J.; Lim, J.; Chang, B. Y.; Lee, H. J.; Chon, B.; Shin, J.; Park, J. B.; Choi, J. H.; Cho, K.; Park, S. M.; Joo, T.; Kim, S. ZnTe/ZnSe (core/shell) type-II quantum dots: their optical and photovoltaic properties. Chem. Mater. 2010, 22, 233240.

(26) Xu, S.; Ziegler, J.; Nann, T. Rapid synthesis of highly luminescent InP and InP/ZnS nanocrystals. J. Mater. Chem. 2008, 18, 2653-2656.

(27) Bahmani Jalali, H.; Karatum, O.; Melikov, R.; Dikbas, U. M.; Sadeghi, S.; Yildiz, E.; Dogru, I. B.; Ozgun Eren, G.; Ergun, C.; Sahin, A.; Kavakli, I. H.; Nizamoglu, S. Biocompatible Quantum Funnels for Neural Photostimulation. Nano Lett. 2019, 19, 5975-5981.

(28) Kumar, B. G.; Sadeghi, S.; Melikov, R.; Aria, M. M.; Jalali, H. B.; Ow-Yang, C. W.; Nizamoglu, S. Structural control of InP/ZnS core/ shell quantum dots enables high-quality white LEDs. Nanotechnology 2018, 29, 345605.

(29) Smith, A. M.; Mohs, A. M.; Nie, S. Tuning the optical and electronic properties of colloidal nanocrystals by lattice strain. Nat. Nanotechnol. 2009, 4, 56-63.

(30) Karatum, O.; Jalali, H. B.; Sadeghi, S.; Melikov, R.; Srivastava, S. B.; Nizamoglu, S. Light-Emitting Devices Based on Type-II InP/ZnO Quantum Dots. ACS Photonics 2019, 6, 939-946.

(31) Shirasaki, Y.; Supran, G. J.; Bawendi, M. G.; Bulović, V. Emergence of colloidal quantum-dot light-emitting technologies. Nat. Photonics 2013, 7, 13-23.

(32) Dai, X.; Zhang, Z.; Jin, Y.; Niu, Y.; Cao, H.; Liang, X.; Chen, L.; Wang, J.; Peng, X. Solution-processed, high-performance light-emitting diodes based on quantum dots. Nature 2014, 515, 96-99.

(33) Liu, J. F.; Bei, Y. Y.; Wu, H. P.; Shen, D.; Gong, J. Z.; Li, X. G.; Wang, Y. W.; Jiang, N. P.; Jiang, J. Z. Synthesis of relatively monodisperse $\mathrm{ZnO}$ nanocrystals from a precursor zinc 2, 4pentanedionate. Mater. Lett. 2007, 61, 2837-2840.

(34) Barak, Y.; Meir, I.; Shapiro, A.; Jang, Y.; Lifshitz, E. Fundamental properties in colloidal quantum dots. Adv. Mater. 2018, 30, 1801442.

(35) Li, L. S.; Pradhan, N.; Wang, Y.; Peng, X. High quality ZnSe and $\mathrm{ZnS}$ nanocrystals formed by activating zinc carboxylate precursors. Nano Lett. 2004, 4, 2261-2264.

(36) Rawalekar, S.; Kaniyankandy, S.; Verma, S.; Ghosh, H. N. Effect of Surface States on Charge-Transfer Dynamics in Type II CdTe/ZnTe Core-Shell Quantum Dots: A Femtosecond Transient Absorption Study. J. Phys. Chem. C 2011, 115, 12335-12342.

(37) Sadeghi, S.; Bahmani Jalali, H.; Srivastava, S. B.; Melikov, R.; Baylam, I.; Sennaroglu, A.; Nizamoglu, S. High-Performance, LargeArea, and Ecofriendly Luminescent Solar Concentrators Using CopperDoped InP Quantum Dots. Iscience 2020, 23, 101272.

(38) Glatter, O. Scattering Methods and Their Application in Colloid and Interface Science; Elsevier, 2018.

(39) Fritz, G.; Glatter, O. Structure and interaction in dense colloidal systems: evaluation of scattering data by the generalized indirect Fourier transformation method. J. Phys.: Condens. Matter 2006, 18, S2403.

(40) Fritz, G.; Bergmann, A. Interpretation of small-angle scattering data of inhomogeneous ellipsoids. J. Appl. Crystallogr. 2004, 37, 815822 .

(41) Ludescher, L.; Dirin, D. N.; Kovalenko, M. V.; Sztucki, M.; Boesecke, P.; Lechner, R. T. Impact of crystal structure and particles 
shape on the photoluminescence intensity of $\mathrm{CdSe} / \mathrm{CdS}$ core/shell nanocrystals. Front. Chem. 2019, 6, 672.

(42) Cantele, G.; Piacente, G.; Ninno, D.; Iadonisi, G. Optical anisotropy of ellipsoidal quantum dots. Phys. Rev. B: Condens. Matter Mater. Phys. 2002, 66, 113308.

(43) van den Broek, M.; Peeters, F. M. Confined states in twodimensional flat elliptic quantum dots and elliptic quantum wires. Phys. E 2001, 11, 345-355.

(44) Lechner, R. T.; Fritz-Popovski, G.; Yarema, M.; Heiss, W.; Hoell, A.; Schülli, T. U.; Primetzhofer, D.; Eibelhuber, M.; Paris, O. Crystal phase transitions in the shell of $\mathrm{PbS} / \mathrm{CdS}$ core/shell nanocrystals influences photoluminescence intensity. Chem. Mater. 2014, 26, 59145922.

(45) Virieux, H.; Le Troedec, M.; Cros-Gagneux, A.; Ojo, W.-S.; Delpech, F.; Nayral, C.; Martinez, H.; Chaudret, B. InP/ZnS nanocrystals: coupling NMR and XPS for fine surface and interface description. J. Am. Chem. Soc. 2012, 134, 19701-19708.

(46) Kazmerski, L. L.; Ireland, P. J.; Sheldon, P.; Chu, T. L.; Chu, S. S.; Lin, C. L. Comparison of low-temperature oxides on polycrystalline InP by AES, SIMS, and XPS. J. Vac. Sci. Technol. 1980, 17, 1061-1066.

(47) Pietra, F.; De Trizio, L.; Hoekstra, A. W.; Renaud, N.; Prato, M.; Grozema, F. C.; Baesjou, P. J.; Koole, R.; Manna, L.; Houtepen, A. J. Tuning the lattice parameter of In X Zn Y P for highly luminescent lattice-matched core/shell quantum dots. ACS Nano 2016, 10, 47544762.

(48) Xi, L.; Cho, D.-Y.; Besmehn, A.; Duchamp, M.; Grützmacher, D.; Lam, Y. M.; Kardynal, B. E. Effect of zinc incorporation on the performance of red light emitting InP core nanocrystals. Inorg. Chem. 2016, 55, 8381-8386.

(49) Granada-Ramirez, D. A.; Arias-Cerón, J. S.; Pérez-González, M.; Luna-Arias, J. P.; Cruz-Orea, A.; Rodríguez-Fragoso, P.; Herrera-Pérez, J. L.; Gómez-Herrera, M. L.; Tomás, S. A.; Vázquez-Hernández, F.; Durán-Ledezma, A. A.; Mendoza-Alvarez, J. G. Chemical synthesis and optical, structural, and surface characterization of InP-In2O3 quantum dots. Appl. Surf. Sci. 2020, 530, 147294.

(50) Mohapatra, P.; Dung, M. X.; Choi, J.-K.; Jeong, S.-H.; Jeong, H.D. Effects of curing temperature on the optical and charge trap properties of InP quantum dot thin films. Bull. Korean Chem. Soc. 2011, 32, 263-272.

(51) Karatum, O.; Eren, G. O.; Melikov, R.; Onal, A.; Ow-Yang, C. W.; Sahin, M.; Nizamoglu, S. Quantum dot and electron acceptor nanoheterojunction for photo-induced capacitive charge-transfer. Sci. Rep. 2021, 11, 2460.

(52) Moulder, J.; Stickle, W.; Sobol, P.; Bomben, K. Handbook of X-ray Photoelectron Spectroscopy: A Reference Book of Standard Spectra for Identification and Interpretation of XPS Data; PerkinElmer Corporation, 1995.

(53) Gao, D.; Zhang, Z.; Fu, J.; Xu, Y.; Qi, J.; Xue, D. Room temperature ferromagnetism of pure $\mathrm{ZnO}$ nanoparticles. J. Appl. Phys. 2009, 105, 113928.

(54) Liang, Y.-C.; Wang, C.-C. Surface crystal feature-dependent photoactivity of $\mathrm{ZnO}-\mathrm{ZnS}$ composite rods via hydrothermal sulfidation. RSC Adv. 2018, 8, 5063-5070.

(55) Choi, Y. I.; Lee, S.; Kim, S. K.; Kim, Y.-I.; Cho, D. W.; Khan, M. M.; Sohn, Y. Fabrication of $\mathrm{ZnO}, \mathrm{ZnS}, \mathrm{Ag}-\mathrm{ZnS}$, and $\mathrm{Au}-\mathrm{ZnS}$ microspheres for photocatalytic activities, $\mathrm{CO}$ oxidation and 2hydroxyterephthalic acid synthesis. J. Alloys Compd. 2016, 675, 46-56.

(56) Kim, T.; Kim, S. W.; Kang, M.; Kim, S.-W. Large-scale synthesis of InPZnS alloy quantum dots with dodecanethiol as a composition controller. J. Phys. Chem. Lett. 2012, 3, 214-218.

(57) Baylam, I.; Cizmeciyan, M. N.; Kakenov, N.; Kocabas, C.; Sennaroglu, A. Ultrafast spectroscopy of voltage reconfigurable graphene saturable absorbers in the visible and near infrared. $2 D$ Materials 2019, 6, 035013.

(58) Zhu, H.; Song, N.; Rodríguez-Córdoba, W.; Lian, T. Wave function engineering for efficient extraction of up to nineteen electrons from one CdSe/CdS quasi-type II quantum dot. J. Am. Chem. Soc. 2012, 134, 4250-4257.
(59) Rawalekar, S.; Kaniyankandy, S.; Verma, S.; Ghosh, H. N. Ultrafast Charge Carrier Relaxation and Charge Transfer Dynamics of $\mathrm{CdTe} / \mathrm{CdS}$ Core- Shell Quantum Dots as Studied by Femtosecond Transient Absorption Spectroscopy. J. Phys. Chem. C 2010, 114, 14601466.

(60) Dutta, A.; Bera, R.; Ghosh, A.; Patra, A. Ultrafast Carrier Dynamics of Photo-Induced Cu-Doped CdSe Nanocrystals. J. Phys. Chem. C 2018, 122, 16992-17000.

(61) Peng, P.; Sadtler, B.; Alivisatos, A. P.; Saykally, R. J. Exciton Dynamics in CdS - Ag2S Nanorods with Tunable Composition Probed by Ultrafast Transient Absorption Spectroscopy. J. Phys. Chem. C 2010, $114,5879-5885$.

(62) Jang, E.; Kim, Y.; Won, Y.-H.; Jang, H.; Choi, S.-M. Environmentally Friendly InP-Based Quantum Dots for Efficient Wide Color Gamut Displays. ACS Energy Lett. 2020, 5, 1316-1327.

(63) Klimov, V.; Hunsche, S.; Kurz, H. Biexciton effects in femtosecond nonlinear transmission of semiconductor quantum dots. Phys. Rev. B: Condens. Matter Mater. Phys. 1994, 50, 8110.

(64) Klimov, V. I.; McBranch, D. W.; Leatherdale, C. A.; Bawendi, M. G. Electron and hole relaxation pathways in semiconductor quantum dots. Phys. Rev. B: Condens. Matter Mater. Phys. 1999, 60, 13740.

(65) Burda, C.; Link, S.; Green, T. C.; El-Sayed, M. A. New transient absorption observed in the spectrum of colloidal CdSe nanoparticles pumped with high-power femtosecond pulses. J. Phys. Chem. B 1999, 103, 10775-10780.

(66) Sadeghi, S.; Ganesh Kumar, B.; Melikov, R.; Mohammadi Aria, M.; Bahmani Jalali, H.; Nizamoglu, S. Quantum dot white LEDs with high luminous efficiency. Optica 2018, 5, 793-802.

(67) McDonald, J. C.; Whitesides, G. M. Poly (dimethylsiloxane) as a material for fabricating microfluidic devices. Acc. Chem. Res. 2002, 35, 491-499.

(68) Cai, D. K.; Neyer, A.; Kuckuk, R.; Heise, H. M. Optical absorption in transparent PDMS materials applied for multimode waveguides fabrication. Opt. Mater. 2008, 30, 1157-1161.

(69) Schubert, E. F.; Gessmann, T.; Kim, J. K. Light emitting diodes. Kirk-Othmer Encyclopedia of Chemical Technology; Wiley, 2000. 